\title{
Illordi/dje Revute.
}

\section{Snternationale Beitidrift}

fïit

Riteratur, faunft und öffentlidbes $\mathfrak{R}$ ebent.

5̧crausgegeben

wง

Dr. wilbalm wolffobn.

Vierter gand.

- $2 x$

瑟eipzig,

$\mathfrak{y} \mathfrak{e}\lfloor\mathfrak{a} \mathfrak{g} \mathfrak{y} \mathfrak{a}$ Carl b. Pord.

1865. 
Untex Aedtsperwabrung gegen HadDrudh und Heberfebung. 